\title{
Optics training programs for primary teachers: using the new Mexican education program
}

Cristina Solano, Charvel López

Cristina Solano, Charvel López Sr., "Optics training programs for primary teachers: using the new Mexican education program," Proc. SPIE 10741, Optics Education and Outreach V, 1074107 (14 September 2018); doi: $10.1117 / 12.2321274$

EDIE Event: SPIE Optical Engineering + Applications, 2018, San Diego, California, United States 


\title{
Optics training programs for primary teachers, using the new Mexican education program
}

\author{
Cristina Solano and Charvel López \\ Centro de Investigaciones en Óptica, A.C. León, Gto., México
}

\begin{abstract}
In recent years the Mexican government has modified the educational programs to include different subjects. Analyzing the natural science books we can notice that in the different units the themes are taught in a direct way introducing in every concept the relation with the everyday life of the students. Optics themes are included in a very good way. The programs some teachers are facing now is that some of them do not have enough experience in this change so a training program has been establish to improve their skills to show them how to implement experiments about the theoretical concepts and to have a wider amount of experiment to enrich the subject.
\end{abstract}

Key words: Formal education, basic optics concepts, optics experiments, outreach workshops

\section{INTRODUCTION}

The basic motivation of our outreach work is to reverse the alarming decline of interest towards science prevalent amongst young people. It has been reported ${ }^{1}$ that, based on the complexity of social, political and technological environment many people have increasing troubles in understanding their surrounding world. This is an actual problem in our region where the automotive industry have a great need of qualified work force in engineering and they need to look for workers from other states.

As a response of this problem, the Mexican government has done a clear and definitive effort to improve the education quality in all levels, with an emphasis in science and technology. The official books have been changed with different subjects with emphasis in possible applications. Optics themes have been included in some extent. Although is a change in the right direction, the problem schools are facing now, is that teachers do not know all the subjects. Because of our experience in outreach activities, some schools have asked us to design workshops that include all the different subjects incorporated in the official curriculum. Our actual efforts are towards to obtain a manual for the teachers that include the correct interpretation of the optics concepts and the design of different experiments to strengthen science instruction on light and optics included in the formal courses.

\section{ANALYSING THE OPTICS CONTENT IN THE NATURAL SCIENCES PRIMARY'S OFFICIAL BOOKS}

The optics contents in the primary books are:

3th Grade

Light characteristics and its importance

4th Grade

Reflection and refraction of light 
6th Grade

Image formation, spherical mirrors, lenses, human eye, pinhole camera, photographic camera, light pollution and solar kitchens

The goals of our work is to present a manual with clear an useful explanations for the teachers that can help them to improve their understanding in key optics related principles, in this way the primary students will received a better information and opens the opportunity of increasing interest in studying optics.

Working with students we have detected that understanding the behavior of light is challenging, not all have a right idea about light nature, its behavior, and many of them express misunderstandings about the conditions that cause light to "bend" when passing through objects. As they have seen laser beams, there is a general idea to think that light rays concretely, represent a physical entities emitted by a source, rather than as abstract, geometric representation used by scientists. Not all primary teachers can correct these ideas as they follow pedagogical studies with out to much science education. The new natural science programs impose a challenge for them and opportunity for us.

We have designing clear experiments in the hope they correct all the misunderstandings giving complementary information for the teachers.

In these work we use previous reported experiences ${ }^{2}$. We are showing here part of the work done analyzing the official books:

In the book corresponding to the 3th grade it is suggested experiment to show that light travels in straight lines is shown in Fig. 1. It is supposed that a hand lamp is used and two pieces of cardboard with holes as shown in the figure; with this set up it is suggested that it is possible to prove that, as the light do not shine the second cardboard, light travels in a straight line, the optical axis. As it is the only experiment suggested in the book, the interpretation is not clear. In any part of the book it is said that if the other holes are illuminated you can find the transmitted light in other position.

In this case, the solution is simple, we do not need to construct a complicated kit, we have just to modify the second paper and correct the interpretation of the book.

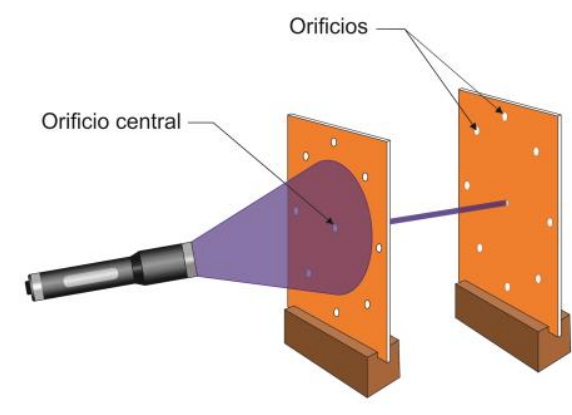

Fig. 1 Suggested experiment to show propagation of light.

4th grade: The book corresponding to the fourth grade, says that the reflection law can be proven with the experiment shown in Fig. 2. In this case a hand lamp is used again, concentrating it with a carbon tube. It is mentioned that all the windows have to be covered to be able to see the reflected light. 
As it is very difficult to obtain clear results with the suggested set up, we recommend the use of a low power laser to increase the visibility of the light. To guaranty the safety of the student we used a laser line lever (3), Class II with a maximum output of $2.2 \mathrm{~mW}$. The experiment was done with more than 300 students; we found out that the use of the laser is very attractive to the children. During the session, all the necessary precautions were made to avoid any accident. All the safety rules are explained and we found that the students follow them without any problem, Fig 3 (a), shows an image of the experiment. The problem with this set up is that the students had problems aligning, placing the beam outside the center of the protractor, as it is shown in Fig. 3 (b).

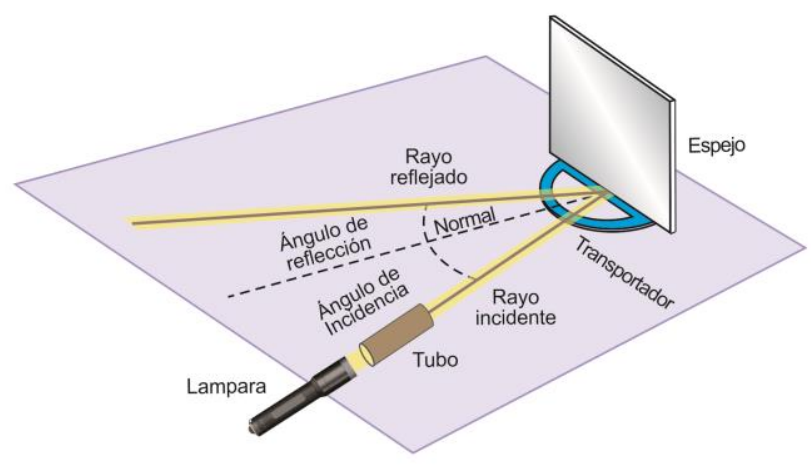

Fig.2 Suggested experiment to show reflection law.
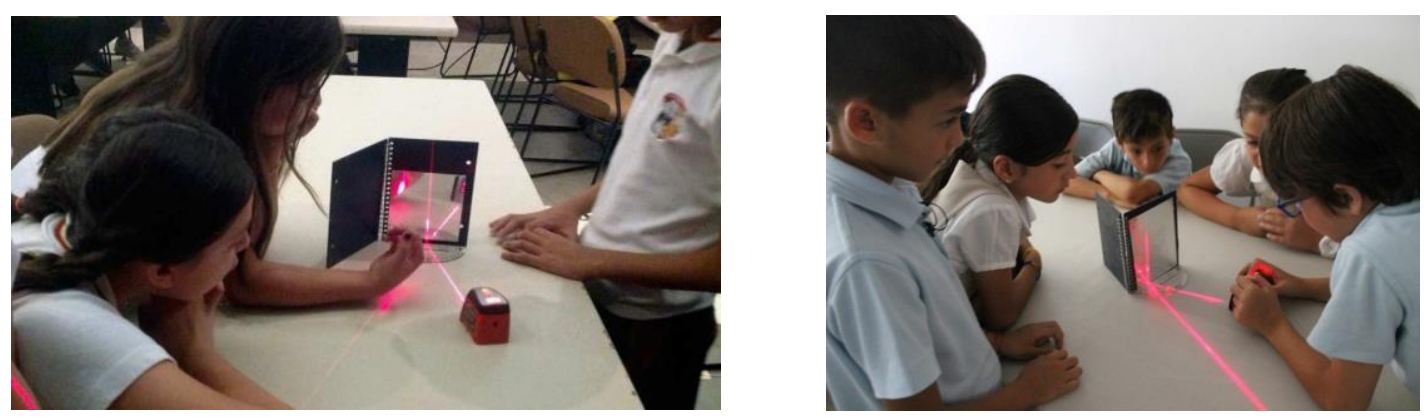

Fig 3. (a) Measuring of the reflection angle, (b) misalignment problems.

To solve this problem the following mount has been designed, Fig. (4), in this set up, the incidence angle of the light can be changed but the position with respect to the center of the mirror is maintain, therefore precise measurements can be done, the protractor is not shown. 


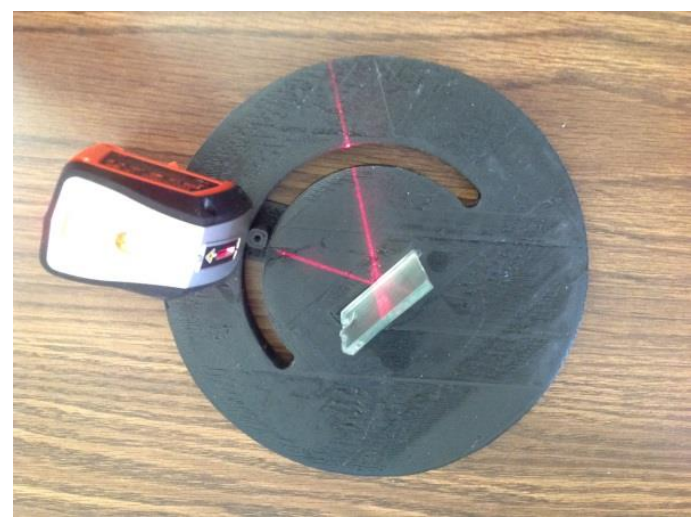

Fig.4. Mount design to prove the reflection law. The protractor is not shown.

The light reflection law can be applied in optical instruments as periscopes. Fig. 5, show the templated designed to ease the constructed of this instrument.

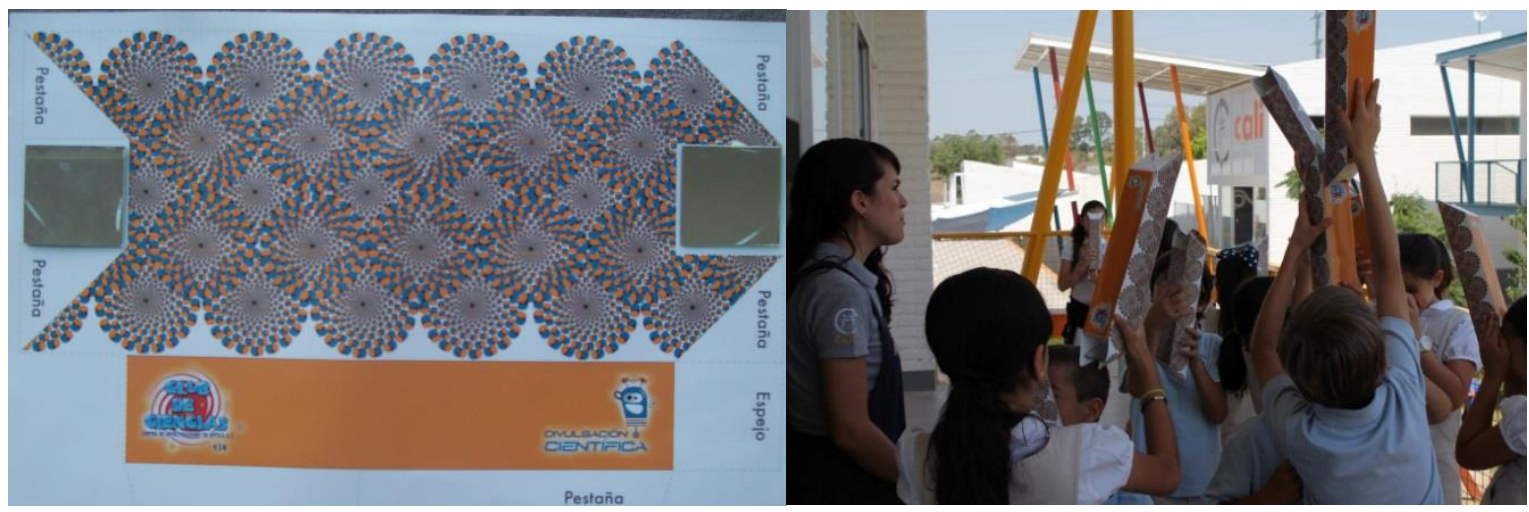

Fig. 5 Template of a periscope

Light refraction: In this case the phenomenon is described by examples, they ask the children to observe a pencil when it is in a glass of water, compared with another one outside it.

It is suggested that the teacher can demonstrate the reflection law in a water tank with a low intensity laser pointer. It is not difficult to cut some glass or acrylic pieces to show how lenses work as in Fig. 6. It is suggested to give the student inexpensive magnifying glasses to observe the light behavior concentrating the light. Another suggestion is to observe the image using one and two magnifying glasses simulating a telescope.

Nevertheless we have found that even after these experiments the students have difficult to understand the image formation so we have designed a camera model with a Fresnel lens, Fig 7. The pieces go one inside the other allowing focusing objects placed at different distances. The final result is shown in Fig. 8. This experiment has proved to be very successful and all students even high school students really enjoy the results. 


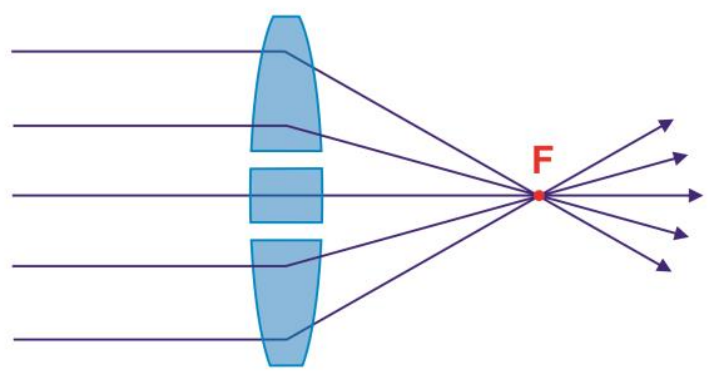

Fig. 6 Simple model of a lens using acrylic or glass pieces as shown

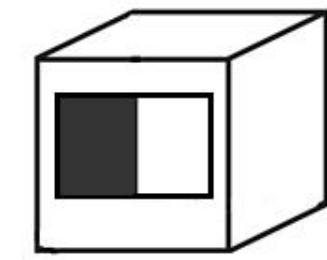

a)

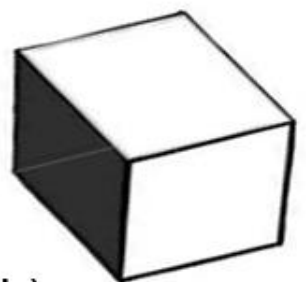

b)

Fig. 7 Designed template for the camera model. a) The front part is covered with the Fresnel lens, in the back of part (b) a translucent paper is placed as a screen.
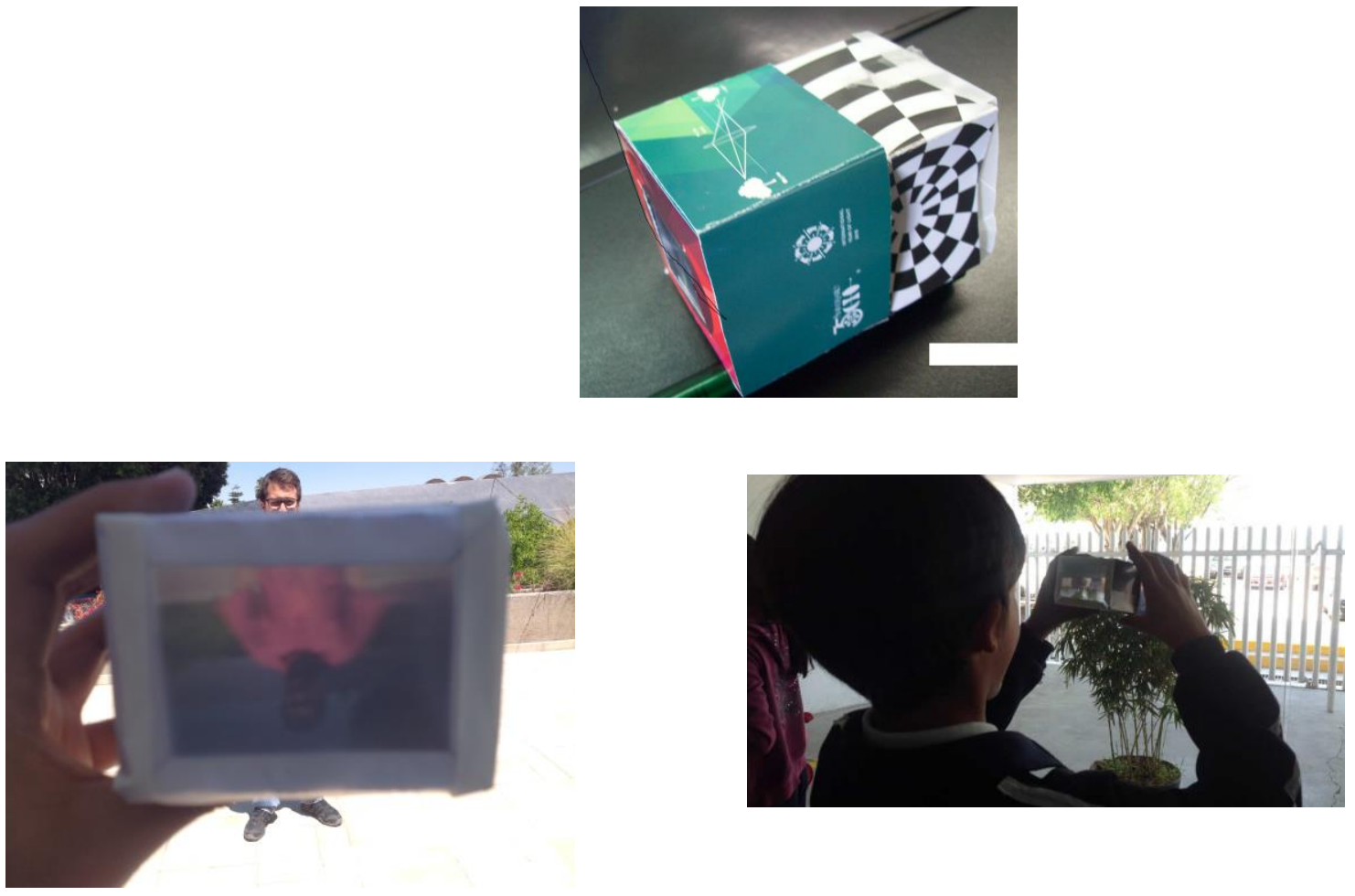

Fig. 8 Camera model, final results and in the photographs the image is clearly seen. 


\section{CONCLUSIONS}

Only some of the work done is show here. We have incorporates our outreach experience to help teachers and students to improve their compression of optics phenomena with a complete experience showing clear and attractive results. A solid foundation of lessons has been establish and it was found that the students are eager to learn and found the use of the laser very attractive.

We plan to start training teachers to improve their optical knowledge, as a means to correct wrong concepts to obtain a faster impact in a larger number of children.

\section{REFERENCES}

[1] Leonard, M.J., Hannahoe, R. M and Nollmeyer, G.E. "Turning Eyes to the Big Sky project: Learning optics in middle school," Proc. SPIE 8481, 84810G-1 (2012)

[2] Magnani, N. Donnelly, M., Mahan, G., Rossi, T., "Lessons learned from student outreach: introducing optics to fifth graders", Proc. SPIE 8481, 848101-1 (2012)

[3] Laser line level, Black and Decker MOD:BDL220S 